\title{
An Augmented Bio-Inspired Algorithm (JA-ABC5a) to Design Optimal Digital IIR Filter
}

\author{
NOORAZLIZA SULAIMAN ${ }^{1}$, JUNITA MOHAMAD-SALEH ${ }^{2 *}$ \\ ABDUL GHANI ABRO ${ }^{3}$, WENG-HOOI TAN ${ }^{2}$ \\ ${ }^{1}$ Faculty of Electrical \& Electronic Engineering Technology, Universiti Malaysia Pahang, 26600 \\ Pekan, Pahang, MALAYSIA \\ ${ }^{2}$ School of Electrical \& Electronic Engineering, Universiti Sains Malaysia, Engineering Campus, \\ 14300 Nibong Tebal, Penang, MALAYSIA \\ ${ }^{3}$ Electrical Engineering Department, NED University of Engineering and Technology, Karachi, \\ PAKISTAN.
}

\begin{abstract}
Among swarm-intelligence based (SI) algorithms, artificial bee colony (ABC) algorithm that is inspired by the intelligent behavior of honeybees has recently captured much interest from optimization researchers. They have come out with the proposal of $\mathrm{ABC}$ variants with the aims to cater the limitations' of $\mathrm{ABC}$; slow convergence speed on unimodal functions and premature convergence tendency on multimodal functions. This research has recently become the main topic among optimization researchers. Nonetheless, the variants also have their limitations as they cannot solve the problem simultaneously. With the motivation from those inefficiencies, this work presents a new modified ABC variant, referred to as JA-ABC5a as a problem solver. JA-ABC5a is simulated on 27 commonly used benchmark functions prior to evaluating its optimization performance. Upon justifying its robustness, it is being applied to design an optimal digital IIR filter.
\end{abstract}

Key-Words: Artificial bee colony, Benchmark functions, Digital IIR filter, Swarm-intelligence algorithm.

Received: September 14, 2020. Revised: December 3, 2020. Accepted: December 19, 2020. Published: December 23, 2020.

\section{Introduction}

Recently, researchers have implemented bioinspired computational intelligence algorithms such as evolutionary programming (EP), evolutionary strategies (ES), particle swarm optimization (PSO) algorithms and bacterial foraging optimization (BFO) with the attempt to efficiently solve complex problems. Those techniques have shown the ability to overcome the limitation of classic techniques by achieving solution closest to the global optimum as well the ability to solve discontinuous and nonconvex objective functions $[1,2]$.

Among them, swarm intelligence-based (SI) algorithms such as firefly algorithm [3], artificial bee colony algorithm [4] and discrete particle swarm optimization [5] have attracted much attention from optimization researchers due to their ability to intelligently perform cooperation among the cooperative agents in their population. Moreover, they demonstrate unique attributes such as self-organized and decentralized control, making them a powerful tool to solve various optimization problems. However, the capabilities of bio-inspired computational intelligence algorithms are still doubted since they are found to be incapable of handling integer and discrete nature problems [1].

Among SI algorithms, the intelligent foraging behavior of honeybees through artificial bee colony (ABC) algorithm has captured much interest from researchers around the world due to its flexibility and simplicity [6]. It has shown better performance than other prominent optimization algorithms such as differential evolution (DE), genetic algorithm (GA), ant colony optimization (ACO) algorithm and particle swarm optimization (PSO) algorithm [6, 7]. Despite that, it faces the problem of slow convergence speed as well as tendency to converge prematurely which is basically due to its mutation equation. Its mutation is known to be good in exploration but poor at exploitation [8-10]. To overcome the problem, researchers have come out with the proposal of $\mathrm{ABC}$ variants. Unfortunately, most of the variants are incapable to counter those problems at once. Motivated from those flaws, this work proposes an augmented $\mathrm{ABC}$ variant named as JA-ABC5a as a vital solution.

Designing optimal digital Infinite impulse response (IIR) filters is known to be a complex task [11]. Nonetheless, they are required in digital signal processing to attenuate and eradicate undesired noise [12]. Besides, they require less computation and use a finite number of variables [13, 14]. They have also been successfully applied to solve various types of applications such as in wireless applications by Joshi, Madanayake [15], grid connected voltage source inverters by Gonzalez-Espin, Patrao [16], and temperature of power electronic device prediction by Davidson, Stone [17]. Due to that, 
researchers have recently paid much attention to designing IIR filters [11].

There are two methods to design a digital IIR filter. One of them is parameter estimation in which an estimation of parameter settings of the filter; first and second-order coefficients, is required to produce a desired digital IIR filter. The estimation is subjected to meet the required condition while minimizing the magnitude response error. Various techniques have been applied by optimization researchers to optimally estimate the parameter settings of the desired filters including genetic algorithm [18], predator prey optimization method [19], particle swarm optimization algorithm [20], cat swarm optimization algorithm [13], teachinglearning based optimization algorithm [21] and twostage ensemble memetic algorithm [11]. However, the algorithms have been found to require more computational time [13].

Thus, the proposed algorithm in this work, JA$\mathrm{ABC} 5 \mathrm{a}$ will be tested to optimally design a digital IIR filter prior to justifying its robustness.

\section{Artificial Bee Colony (ABC) Algorithm}

Artificial bee colony (ABC) algorithm is proposed by Karaboga in 2005 . It simulates the intelligent foraging behavior of honeybees. It consists of five phases which are initialization, employed-bees, onlooker-bees, scout-bee and termination phases. $\mathrm{ABC}$ algorithm works from the interaction among the bees in three performance-deciding phases which are employed-bees, onlooker-bees and scoutbee phases. The other phases are just supporting phases. The flowchart of the standard ABC is illustrated in Fig. 1.

The algorithm starts with the random initialization of the food sources based on the population-size in the initialization phase. The food sources represent the possible solutions and the number of food sources (i.e. FoodNumber) is basically half of the population size. Next, those food sources are randomly assigned to the employed-bees around the hive. After that, the nectar amount that represents the fitness values of each of the food sources is calculated. In the employed-bees phase, the neighborhood of food sources associated with the employed-bees is explored using the following mutation equation [10]:

$$
z_{i j}=y_{i j}+\phi_{i j}\left(y_{i j}-y_{k j}\right)
$$

where: $z_{i j}=$ New candidate solution of new food sources

$y_{i j}=$ Food source to be updated

$y_{k j}=$ Neighborhood food source

$\phi_{i j}=$ Random numbers in the range $[-1,1]$

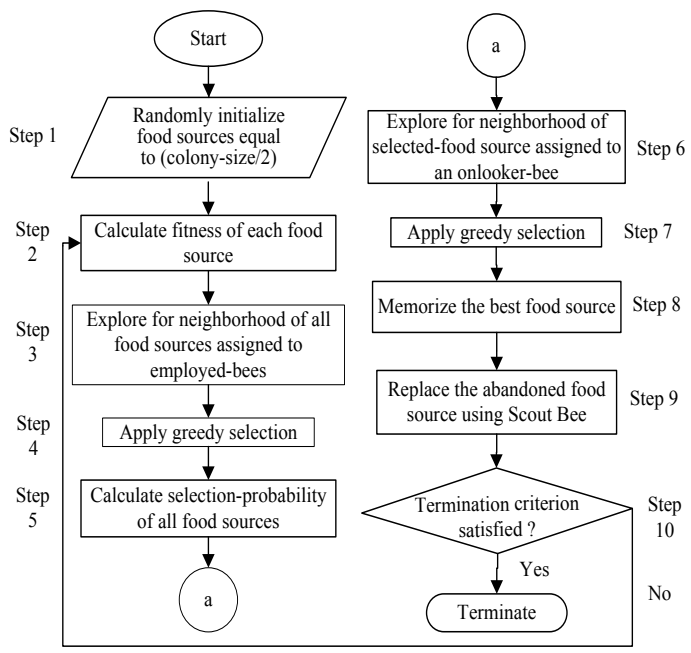

Fig. 1: The flowchart of the standard ABC algorithm

The equation governs the interaction among the food sources and generates new food sources (i.e. candidate solutions). Then, greedy-selection mechanism is employed to select the best food sources between the candidate solution and the old food source $y_{i}$. The best food source among them (i.e. fitter fitness value) is called potential fitter food source. This potentially fitter food sources are then shared with onlooker-bees in the onlooker-bees phase.

In the onlooker-bees phase, the onlooker-bees do not update all potential food sources shared by employed-bees. They are further selected by the onlooker-bees to be updated using fitnessproportion selection scheme that is based on the probability value, $P_{\mathrm{i}}$ associated with the food source. Similar to employed-bees phase, greedy-selection is again applied to choose the best food source. Then, this best food source so far for that generation is memorized.

In the scout-bee phase, the food source that does not show improvement over a certain number of preset generations called limit is to be abandoned [22]. The employed-bees associated with the abandoned food source become scout-bee and will search the environment randomly for discovering a new food source to replace the abandoned food source. This is done in order to balance out the number of food sources in the population. Scout-bee searches the entire search space and picks the replacement using the following equation [10]. 


$$
y_{i}^{j}=y_{\min }^{j}+\operatorname{rand}(0,1)\left(y_{\max }^{j}-y_{\text {min }}^{j}\right)
$$

where: $y_{\min }^{\mathrm{j}}=$ Lower limit of the search space $\mathrm{y}^{\mathrm{j}}$ max $=$ Upper limit of the search space rand $(0,1)=$ Random numbers within $[0,1]$

In the termination phase, the algorithm checks for the termination criterion, maximum-generations. If the termination criterion is not met, then it repeats from step 2 until the numbers are reached. More details on the standard $\mathrm{ABC}$ algorithm can be found in [23] and [10].

\section{Augmented ABC Variant (JA- ABC5A)}

It has been previously mentioned that $\mathrm{ABC}$ has three performance-deciding phases. Thus, any modifications need to be implemented to these phases for effective improvement. Prior to enhance the performance of $\mathrm{ABC}$ and to cater the aroused problems, this research work extends the work by Sulaiman et al. [24] by modifying their proposed variant, JA-ABC5.

It is known that JA-ABC5 is developed by implementing few modifications to the standard $\mathrm{ABC}$ algorithm. JA-ABC5 is said to have balanced exploration and exploitation capabilities as it is able to enhance its exploration capability in the employed-bees phase and improve its exploitation capability in the onlooker-bees phase. However, further analysis has shown that JA-ABC5 still lacks in exploitation as the incorporated equation in the employed-bees phase of JA-ABC5 is known for its randomness [24].

Hence, with the objective to further balance the exploration and exploitation capabilities of the algorithm for robust optimization performance, few modifications need to be done to the existing JAABC5. The modified ABC variant is named as JA$\mathrm{ABC} 5 \mathrm{a}$. The working procedure of JA-ABC5a is illustrated in Fig. 2 with the modified stage highlighted. The modification to JA-ABC5 is done in the employed-bees phase by step 5 given by:

$$
z_{i j}=y_{\text {best }, j}+\phi_{i j}\left(y_{i j}-y_{r l, j}\right)+\psi_{i j}\left(y_{r 2, j}-y_{\text {best }, j}\right)
$$

where: $z_{i j}=$ New candidate solution of new food sources

$y_{\text {best }, j}=$ Global best food source with $j$-th dimension $y_{r l, j}=$ Neighborhood food source $y_{r 2, j}=$ Neighborhood food source

$\phi_{i j}=$ Random numbers in the range $[-1,1]$

$\psi_{i j}=$ Random number in the range of $[0, \mathrm{~T}]$

This equation is generated from hybridizing the mutation equations in the works of Gao and Liu [25], and Abro [26]. The first and third terms of the equation aim to enhance the exploitation capabilities of JA-ABC5a by exploiting the information of the best food source in the current population. This should enhance the convergence rate of JA-ABC5a. Nonetheless, intense exploitation might trap the algorithm in a local optimum, particularly when solving a multimodal function.

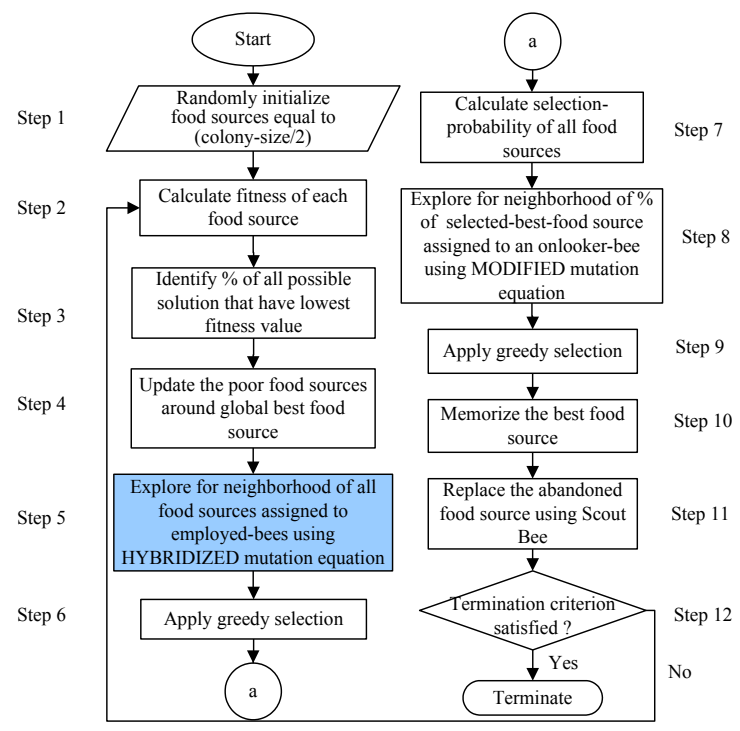

Fig. 2: The flowchart of JA-ABC5a

Thus, here comes the second term of the equation that uses the information of the randomly explored neighborhood food sources. As a result, this should improve the exploitation capability of the algorithm. Moreover, the equation from work [22] is known for its randomness. JA-ABC5a should now possess balanced exploration and exploitation processes and hence should be able to converge faster at achieving the global optimum.

\section{Digital IIR Filter Design}

The main task of designing optimal digital IIR filter is to estimate the settings of the first and secondorder coefficients of the filters given by $a_{0} \ldots a_{n}$, $\mathrm{b}_{0 \ldots} \ldots \mathrm{b}_{n}, c_{11 \ldots} c_{1 m}, c_{21 \ldots} \ldots c_{2 m}, d_{11 \ldots} d_{1 m}$ and $d_{21 \ldots} d_{2 m}$ that would give the minimum values of magnitude response errors on both pass-band and stop-band of the designed filters subjected to their constraints. The mathematical expression of the problem is given by [11]:

$$
\begin{aligned}
\min f_{1} & =\frac{1}{P_{n}} \sum_{i=1}^{P_{n}} H_{p}\left(\omega_{i}\right)+\frac{1}{S_{n}} \sum_{j=1}^{S_{n}} H_{s}\left(\omega_{j}\right) \\
H_{p}(\omega) & = \begin{cases}1-\delta_{1}-\mid H\left(e^{j \omega}\right), & \mid H\left(e^{j \omega}\right)<1-\delta_{1} \\
0, & \left|H\left(e^{j \omega}\right)\right| \geq 1-\delta_{1}\end{cases}
\end{aligned}
$$




$$
\begin{aligned}
& H_{s}(\omega)= \begin{cases}\left|H\left(e^{j \omega}\right)\right|-\delta_{2}, & \left|H\left(e^{j \omega}\right)\right| \geq \delta_{2} \\
0, & \left|H\left(e^{j \omega}\right)\right| \leq \delta_{2}\end{cases} \\
& H\left(e^{j \omega}\right)=K \prod_{k=1}^{n} \frac{1+b_{k}\left(e^{j \omega}\right)^{-1}}{1+a_{k}\left(e^{j \omega}\right)^{-1}} \prod_{i=1}^{m} \frac{1+d_{1 i}\left(e^{j \omega}\right)^{-1}+d_{2 i}\left(e^{j \omega}\right)^{-2}}{1+c_{1 i}\left(e^{j \omega}\right)^{-1}+c_{2 i}\left(e^{j \omega}\right)^{-2}} \\
& -1<a_{k}<1 \\
& -1<b_{k}<1 \\
& -1<c_{i 1}<0 \\
& -1<c_{i 2}<1 \\
& -2<d_{i 1}<2 \\
& -3<d_{i 2}<1
\end{aligned}
$$

where: $P_{n}=$ Sampling frequency in the pass-band

$S_{n}=$ Sampling frequency in the stop-band $H_{p}=$ Pass-band magnitude response error at $\omega$

$H_{s}=$ Stop-band magnitude response error at $\omega$

$\mathrm{K}=$ Filter gain

$a_{k}$ and $b_{k}=$ First-order coefficients

$c_{1 i}, c_{2 i}, d_{1 i}$ and $d_{2 i}=$ Second-order coefficients

Based on the above mathematical expression, the optimal values of $a_{n} a_{0 \ldots} a_{n}, \mathrm{~b}_{0} \ldots \mathrm{b}_{n}, c_{11 \ldots} c_{1 m}, c_{21 \ldots} c_{2 m}$, $d_{11 \ldots} d_{1 m}$ and $d_{21 \ldots} d_{2 m}$ that give the minimum values of magnitude response error of both pass-band and stop-band of the designed filters shall be determined, subjected to the limit of each of the parameters.

\section{Experimental Settings}

The performance of the proposed augmented ABC variant, JA-ABC5a is simulated and analyzed in two ways; its performance on benchmark functions and on the design of optimal digital IIR filters. The general parameters are population-size, maximumgenerations and limit. They have been set to 50, 100 and FoodNumber x $D$, respectively. The FoodNumber represents the size of the possible solution by which indicating half of the populationsize.

\subsection{Benchmark Functions}

The proposed JA-ABC5a has been simulated on 27 various commonly used benchmark functions found in the literature. They are ranged from unimodal to multimodal and from shifted to rotated functions and as listed in Table 1.

The performances of JA-ABC5a on the benchmark functions are compared with the standard $\mathrm{ABC}$ algorithm and existing variants found

\begin{tabular}{|c|c|c|}
\hline $\begin{array}{c}\text { Functio } \\
n\end{array}$ & Function Name & $\begin{array}{c}\text { Initializati } \\
\text { on Range }\end{array}$ \\
\hline$f 1$ & Griewank & \pm 600 \\
\hline$f 2$ & Rastrigin & \pm 15 \\
\hline$f 3$ & Rosenbrock & \pm 15 \\
\hline$f 4$ & RS Ackley & \pm 32 \\
\hline$f 5$ & Schwefel & \pm 500 \\
\hline f6 & Himmelblau & \pm 600 \\
\hline f7 & RS Sphere & \pm 600 \\
\hline$f 8$ & Step & \pm 600 \\
\hline f9 & Bohachevsky 2 & \pm 100 \\
\hline f10 & RS Schwefel 2.22 & \pm 100 \\
\hline f11 & RS Schwefel Ridges & \pm 100 \\
\hline$f 12$ & $\begin{array}{c}\text { RS Schwefel Ridges with } \\
\text { Noise }\end{array}$ & \pm 15 \\
\hline f13 & RS Elliptic & \pm 100 \\
\hline f14 & Zekhelip & \pm 15 \\
\hline f15 & Non-continuos Rastrigin & \pm 15 \\
\hline f16 & Michalewicz & $0-180$ \\
\hline$f 17$ & First Expanded Function & \pm 15 \\
\hline f18 & Second Expanded Function & \pm 15 \\
\hline f19 & Third Expanded Function & \pm 15 \\
\hline$f 20$ & Fourth Expanded Function & \pm 500 \\
\hline$f 21$ & Fifth Expanded Function & \pm 100 \\
\hline$f 22$ & Sixth Expanded Function & \pm 100 \\
\hline$f 23$ & Seventh Expanded Function & \pm 15 \\
\hline$f 24$ & Eighth Expanded Function & \pm 100 \\
\hline$f 25$ & Rotated Griewank Function & {$[0,600]$} \\
\hline$f 26$ & Rotated Ackley Function & \pm 32 \\
\hline$f 27$ & Rotated Rastrigin Function & \pm 5 \\
\hline
\end{tabular}
in the literature in order to validate its performances. The variants include improved $\mathrm{ABC}$ (IABC) [25], global best $\mathrm{ABC}$ (BABC1) [27], enhanced probability-selection ABC (EPS-ABC) [28] and two recently proposed variants; JA-ABC3 [29] and JAABC5 [24] algorithms.

Table 1: Benchmark Functions

\begin{tabular}{|c|c|}
\hline Parameters & Settings \\
\hline Pass-band $(\omega)$ & $0-0.3 \pi \cup 0.7 \pi$ \\
\hline Stop-band $(\omega)$ & $0.4 \pi-0.6 \pi$ \\
\hline$\delta 1(\mathrm{~dB})$ & 1 \\
\hline$\delta 2(\mathrm{~dB})$ & 90 \\
\hline Order & 14 \\
\hline
\end{tabular}

\subsection{Designing Optimal Digital IIR Filter}

The digital IIR filter that will be designed in this work is band-stop (BS) filter. The settings of the filter follow the values from [11] for comparison purpose and are tabulated in Table 2.

Table 2: The Settings of the Designed IIR Filters

The performance of JA-ABC5a is then compared with the same $A B C$ variants as the previous section and other optimization algorithms that have been applied to solve optimal digital IIR filters design found in the work of [11]. They are ensemble 
memetic algorithm (TSMA), modified differential evolution (MDE), self-adaptive differential evolution (SaDE) and local version of particle swarm optimization with constriction factor (PSOcf).

\section{Results \& Discussion}

\subsection{Performance on Benchmark Functions}

As mentioned beforehand, the proposed JA-ABC5a is simulated on 27 commonly used benchmark functions and its performances are then compares with the standard $\mathrm{ABC}$ and three existing $\mathrm{ABC}$ variants. Their performances are evaluated based on the minimum values obtained in 30 runs. The comparison is done on the basis of convergence speed and global optimum achievement. The obtained performances results are as illustrated in Fig. 3 to 7 .

Fig. 4 to 7 show the efficiency of the proposed JA-ABC5a to reach the global optimum in less number of generations compared to the existing variants.

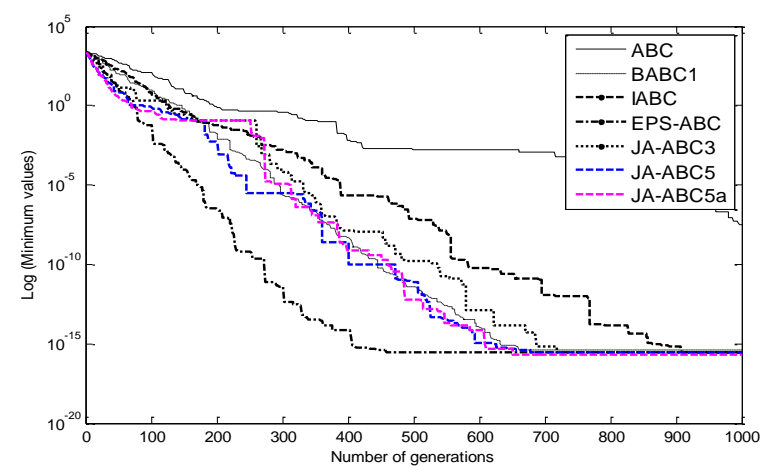

Fig. 3: The performance results of $A B C$ variants on Zekhelip function

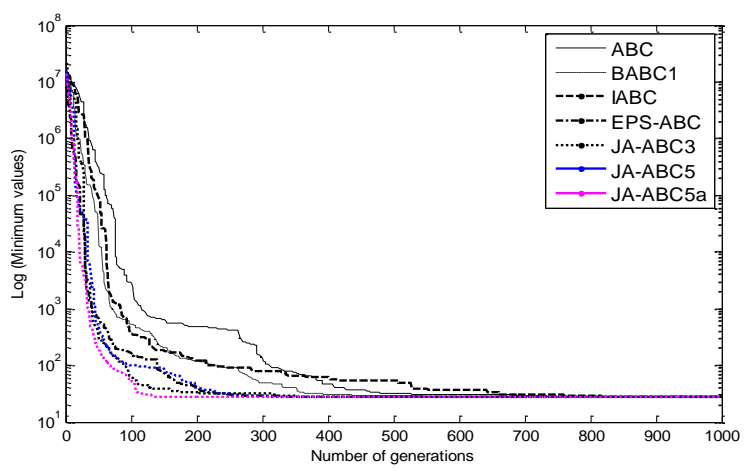

Fig. 4: The performance results of $\mathrm{ABC}$ variants on Rosenbrock plus step function

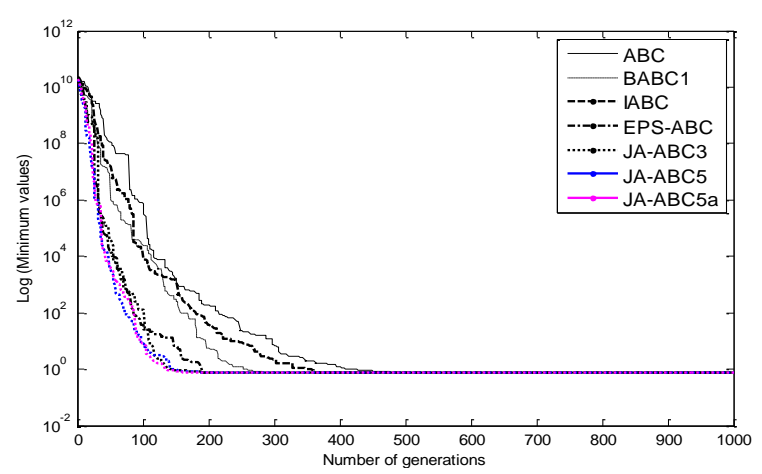

Fig. 5: The performance results of $A B C$ variants on Dixon-Price plus Schwefel Ridge function

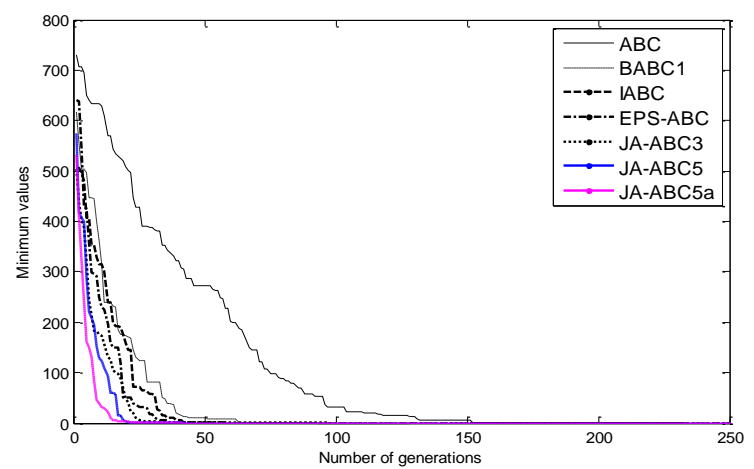

Fig. 6: The performance results of $\mathrm{ABC}$ variants on rotated Griewank function

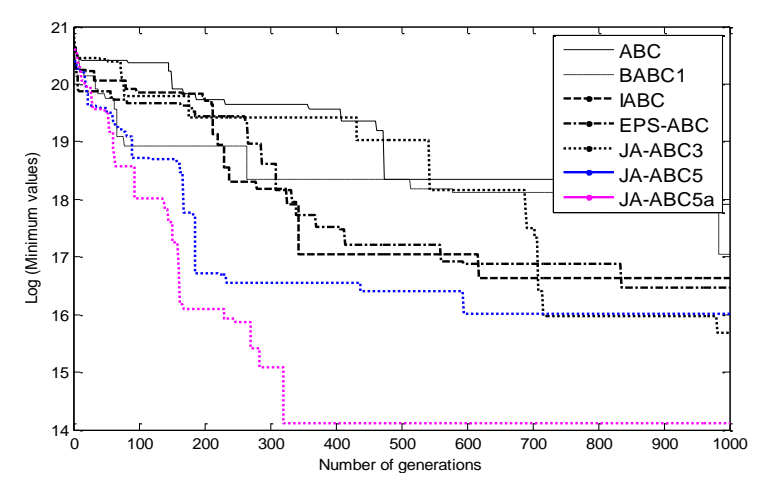

Fig. 7: The performance results of $A B C$ variants on rotated Ackley function

For instance, Fig. 4 illustrates that JA-ABC5a converges faster than the compared variants to reach the global optimum on the expanded function of Rosenbrock and Step functions. JA-ABC5a also seems to converge faster than JA-ABC5 which indicates that the enhancement done to JA-ABC5 in the form of JAABC5a is able to improve its performance. Similarly, Fig. 5 and Fig. 6 also portray the ability of JA-ABC5a to converge the fastest in achieving the global optimum on expanded function of Dixon-Price and Schwefel Ridge functions and rotated Griewank function. 
Meanwhile, Fig. 7 demonstrates that not only JA-ABC5a is the fastest to converge but it is the only algorithm that is able to reach the global optimum on rotated Ackley function. In the meantime, the other compared variants are trapped in the local optima of the function.

On the other hand, Fig. 3 shows the inefficiency of JA-ABC5a to converge the fastest on Zekhelip function. It converges slower than one of the compared variant which is EPS-ABC [28]. Despite that, it basically converges faster than the other compared variants. More importantly, JA-ABC5a reaches the lowest minimum value i.e. global optimum of the function.

The excellent performances shown by JA$\mathrm{ABC} 5 \mathrm{a}$ on the tested benchmark functions vividly prove that the enhancement done to JA-ABC5 has been able to improve its optimization performance. The introduction of new mutation equation by hybridizing two mutation equations in employedbees phase has balanced out both exploration and exploitation of the algorithm. The result is the ability of JA-ABC5a to be able to escape from local minima traps while rapidly converging. The incorporated modifications obviously show that balanced exploration and exploitation capabilities are essential and should be achieved by an optimization algorithm to exhibit robust optimization performance.

\subsection{Designing Optimal Digital IIR Filter}

Once the efficiency of JA-ABC5a as an optimization algorithm has been proven, it is now ready to be applied to design optimal digital IIR filter. It has been applied to estimate the optimal settings of a band-stop (BS) filter. Its performance is being compared with few optimization algorithms as mentioned in the previous section. The acquired results are depicted in Table 3, showing the magnitude response errors produced by the proposed $\mathrm{JA}-\mathrm{ABC} 5 \mathrm{a}$ algorithm, other $\mathrm{ABC}$ variants and other optimization algorithms.

The acquired results clearly demonstrate robust competency of JA-ABC5a to produce minimum value of magnitude response error in comparison to its counterparts. This is particularly due to the amendments made to the standard $\mathrm{ABC}$ algorithm to produce $\mathrm{JA}-\mathrm{ABC} 5 \mathrm{a}$, a modified $\mathrm{ABC}$ variant. The proposed variant has been able to optimize the design of digital IIR filter, a complex real-world application. Additionally, the results in Table 3 show that the existing $\mathrm{ABC}$ variants, including the standard $\mathrm{ABC}$ perform better than the others when they manage to obtain smaller minimum magnitude response error compared to other types of algorithms. This vividly suggest the robustness of $\mathrm{ABC}$ and its variants at solvingral-world optimization problems.

Table 3: Performance results of the optimization algorithms at optimizing BS filter

\begin{tabular}{cc}
\hline Algorithms & Magnitude Response Error \\
TSMA & $3.28 \mathrm{E}+01$ \\
MDE & $1.96 \mathrm{E}+03$ \\
SaDE & $3.87 \mathrm{E}+01$ \\
PSO-cf & $1.51 \mathrm{E}+02$ \\
ABC & $2.35 \mathrm{E}+00$ \\
BABC1 & $2.31 \mathrm{E}+00$ \\
IABC & $2.30 \mathrm{E}+00$ \\
EPS-ABC & $2.34 \mathrm{E}+00$ \\
JA-ABC3 & $2.31 \mathrm{E}+00$ \\
JA-ABC5 & $2.30 \mathrm{E}+00$ \\
JA-ABC5a & $\mathbf{2 . 2 9 E}+\mathbf{0 0}$ \\
\hline
\end{tabular}

\section{Conclusion}

This paper proposes the development of an augmented $\mathrm{ABC}$ variant named as JA-ABC5a. It is formulated by modifying the recently proposed variant, JA-ABC5 prior to enhance and balance its exploration and exploitation capabilities. This is because a robust optimization algorithm should have balanced of those processes. The result is an augmented $\mathrm{ABC}$ variant in form of JA-ABC5a that has the fastest convergence rates in reaching the global optimum. This is portrayed by the graphical results. JA-ACB5a converges the fastest among all compared variants including the recently proposed variants (i.e. JA-ABC3 and JA-ABC5) on numbers of tested benchmark functions. There are few cases in which JA-ABC5a show slower convergence than EPS-ABC, but it actually converges faster than the others. Moreover, the most important thing to mention is JA-ABC5a is able to reach the global optimum when the other algorithms have been trapped in the functions' local minima. The efficiency of JA-ABC5a is further demonstrated as it has successfully optimized the setting of BS filter by producing the least magnitude response error. The results vividly verify the robustness of JA$\mathrm{ABC} 5 \mathrm{a}$ as an optimization algorithm that is able to solve the complex real-world application.

\section{References:}

[1] Ayan, K. and U. K1lıç, Artificial bee colony algorithm solution for optimal reactive power 
flow. Applied Soft Computing, 2012. 12(5): p. 1477-1482.

[2] Mehdinejad, M., et al., Solution of optimal reactive power dispatch of power systems using hybrid particle swarm optimization and imperialist competitive algorithms. International Journal of Electrical Power \& Energy Systems, 2016. 83: p. 104-116.

[3] Bacanin, N. and M. Tuba, Firefly Algorithm for Cardinality Constrained Mean-Variance Portfolio Optimization Problem with Entropy Diversity Constraint. The Scientific World Journal, 2014: p. 16.

[4] Slimani, L. and T. Bouktir, Economic power dispatch of power systems with pollution control using artificial bee colony optimization. Turkish Journal of Electrical Engineering \& Computer Sciences, 2013. 21: p. 1515-1527.

[5] Sevkli, A.Z. and F.E. Sevilgen, Discrete particle swarm optimization for the team orienteering problem. Turkish Journal of Electrical Engineering \& Computer Sciences, 2012. 20(231-239).

[6] Karaboga, D. and B. Basturk, On the performance of artificial bee colony $(A B C)$ algorithm. Applied Soft Computing, 2008. 8(1): p. 687-697.

[7] Karaboga, D. and B. Basturk, A powerful and efficient algorithm for numerical function optimization: artificial bee colony $(A B C)$ algorithm. Journal of Global Optimization, 2007. 39(3): p. 459-471.

[8] Abro, A.G. and J. Mohamad-Saleh. Enhanced Global-Best Artificial Bee Colony Optimization Algorithm. in Computer Modeling and Simulation (EMS), 2012 Sixth UKSim/AMSS European Symposium on. 2012. Valetta.

[9] Abro, A.G. and J. Mohamad-Saleh, An Enhanced Artificial Bee Colony Optimization Algorithm. Recent Advances in Systems Science and Mathematical Modelling, ed. D.S. Nikos Mastorakis, Valeriu Prepelita. 2012: WSEAS Press.

[10] Karaboga, D. and B. Akay, A comparative study of Artificial Bee Colony algorithm. Applied Mathematics and Computation, 2009. 214(1): p. 108-132.

[11] Wang, Y., B. Li, and T. Weise, Two-stage ensemble memetic algorithm: Function optimization and digital IIR filter design. Information Sciences, 2013. 220(0): p. 408424.

[12] Pan, S.-T., Evolutionary Computation on Programmable Robust IIR Filter PolePlacement Design. IEEE Transactions on
Instrumentation and Measurement 2011. 60(4): p. 1469-1479.

[13] Panda, G., P.M. Radhan, and B. Majhi, IIR system identification using cat swarm optimization. Expert Systems with Applications, 2011. 38(10): p. 12671-12683.

[14] Tsai, J.-T., W.-H. Ho, and J.-H. Chou, Design of two-dimensional IIR digital structurespecified filters by using an improved genetic algorithm. Expert Systems with Applications, 2009. 36(3, Part 2): p. 6928-6934.

[15] Joshi, R.M., et al., Synthesis and Array processor Realization of a 2-D IIR Beam Filter for Wireless Applications. IEEE Transactions on Very Large Scale Integration (VLSI) Systems, 2012. 20(12): p. 2241-2254.

[16] Gonzalez-Espin, F., et al., An Adaptive Digital Control Technique for Improved Performance of Grid Connected Inverters. IEEE Transactions on Industrial Informatics, 2013. 9(2): p. 708-718.

[17] Davidson, J.N., D.A. Stone, and M.P. Foster, Real-Time Prediction of Power Electronic Device Temperatures Using PRBS-Generated Frequency-Domain Thermal Cross Coupling Characteristics. IEEE Transactions on Power Electronics, 2015. 30(6): p. 2950-2961.

[18] Yang, $\mathrm{Y}$. and $\mathrm{X} . \mathrm{Yu}$, Cooperative Coevolutionary Genetic Algorithm for Digital IIR Filter Design. IEEE Transactions on Industrial Electronics, 2007. 54(3): p. 13111318.

[19] SINGH, B., DHILLON, J. S., \& BRAR, Y. S. (2013). Predator Prey Optimization Method For The Design Of IIR Filter. WSEAS TRANSACTIONS ON SIGNAL PROCESSING, 9(2), P. 51-62.

[20] SINGH, D., \& DHILLON, J. S. (2015). Design of optimal IIR digital filter using TeachingLearning based optimization technique. WSEAS TRANSACTIONS on ADVANCES in ENGINEERING EDUCATION, 12, P. 9-18.

[21] SUGITA, Y. (2016). Design of IIR filters with reduced group delay ripple via particle swarm optimization. WSEAS TRANSACTIONS on SIGNAL PROCESSING, 12, P. 192-198. 
[22] Zhu, G. and S. Kwong, Gbest-guided artificial bee colony algorithm for numerical function optimization. Applied Mathematics and Computation, 2010. 217(7): p. 3166-3173.

[23] Karaboga, D., An Idea Based on Honey Bee Swarm For Numerical Optimization. Technical Report-TR06, 2005.

[24] Sulaiman, N., J. Mohamad-Saleh, and A.G. Abro, New Enhanced Artificial Bee Colony (JA-ABC5) Algorithm with Application for Reactive Power Optimization. The Scientific World Journal, 2015, article ID 396189, 2015.

[25] Gao, W. and S. Liu, Improved artificial bee colony algorithm for global optimization. Information Processing Letters, 2011. 111(17): p. 871-882.

[26] Abro, A.G., Performance Enhancement of Artificial Bee Colony Optimization Algorithm, in School of Electrical \& Electronic Engineering, 2013, Universiti Sains Malaysia: Malaysia

[27] Gao, W., S. Liu, and L. Huang, A global best artificial bee colony algorithm for global optimization. Journal of Computational and Applied Mathematics, 2012. 236(11): p. 27412753.

[28] Abro, A.G. and J. Mohamad-Saleh, Enhanced probability-selection artificial bee colony algorithm for economic load dispatch: A comprehensive analysis Engineering Optimization, 2014. 46(10): p. 1315-1330.

[29] Sulaiman, N., J. Mohamad-Saleh, and A.G. Abro, Modified artificial bee colony algorithm for reactive power optimization. AIP Conference Proceedings, 2015. 1660(1): p. 050037.

\section{Contribution of individual authors}

Noorazliza Sulaiman, implemented the algorithm, carried out the simulation and optimization, and prepared the manuscript.

Junita Mohamad-Saleh, responsible for mentorship to the team, research activity planning and execution, and manuscript's review for content as well as English editing.

Abdul Ghani Abro, contributed ideas and original code.

Weng Hooi Tan, prepared the final paper into required template, and reviewed the manuscript.

\section{Sources of funding for research presented in a scientific article or scientific article itself \\ Source of funding: MOHE FRGS Grant Number: RDU190180.}

\section{Creative Commons Attribution License 4.0 (Attribution 4.0 International, CC BY 4.0)}

This article is published under the terms of the Creative Commons Attribution License 4.0 https://creativecommons.org/licenses/by/4.0/deed.en_US 